Article

\title{
Direct Growth of Antimonene on C-Plane Sapphire by Molecular Beam Epitaxy
}

\author{
Minghui Gu ${ }^{1}$, Chen Li ${ }^{1}$, Yuanfeng Ding ${ }^{1}$, Kedong Zhang ${ }^{1}$, Shunji Xia ${ }^{1}$, Yusheng Wang ${ }^{1}$, \\ Ming-Hui Lu ${ }^{1,2}$, Hong Lu 1,2,*D and Yan-Feng Chen ${ }^{1}$ \\ 1 National Laboratory of Solid State Microstructures \& Department of Materials Science and Engineering, \\ College of Engineering and Applied Sciences, Nanjing University, Nanjing 210093, Jiangsu, China; \\ gmh309780480@163.com (M.G.); chenli@nju.edu.cn (C.L.); yfding_nju@163.com (Y.D.); \\ njzkd012@163.com (K.Z.); 141190089@smail.nju.edu.cn (S.X.); wang15062261565@163.com (Y.W.); \\ luminghui@nju.edu.cn (M.-H.L.); yfchen@nju.edu.cn (Y.-F.C.) \\ 2 Jiangsu Key Laboratory of Artificial Functional Materials, Nanjing 210093, Jiangsu, China \\ * Correspondence: hlu@nju.edu.cn
}

Received: 8 November 2019; Accepted: 10 January 2020; Published: 16 January 2020

Featured Application: Two-dimensional (2D) featured antimonene has been directly grown on sapphire substrates by molecular beam epitaxy. The van der Waals epitaxy growth can be promoted through temperature control in the growth window. The competing mechanism between $2 \mathrm{D}$ and three-dimensional (3D) growths and the effects of adsorption and cracking of the source molecules are discussed.

\begin{abstract}
Monolayer antimony (antimonene) has been reported for its excellent properties, such as tuneable band gap, stability in the air, and high mobility. However, growing high quality, especially large-area antimonene, remains challenging. In this study, we report the direct growth of antimonene on c-plane sapphire substrate while using molecular beam epitaxy (MBE). We explore the effect of growth temperature on antimonene formation and present a growth phase diagram of antimony. The effect of antimony sources $\left(\mathrm{Sb}_{2}\right.$ or $\left.\mathrm{Sb}_{4}\right)$ and a competing mechanism between the two-dimensional (2D) and three-dimensional (3D) growth processes and the effects of adsorption and cracking of the source molecules are also discussed. This work offers a new method for growing antimonene and it provides ideas for promoting van der Waals epitaxy.
\end{abstract}

Keywords: antimonene; molecular beam epitaxy; effective adsorption; sapphire; two dimensional materials

\section{Introduction}

Nowadays, two-dimensional (2D) materials are popular in materials science research as a rising star after Geim put forward the concept of graphene [1]. People have put great amount of efforts to make large area graphene for practical applications. There have been some successes in growing large area and high-quality graphene films by ultrafast growth techniques [2,3]. However, because of limitations, such as the zero band gap, the broad application of graphene is still challenging. Transition-metal dichalcogenides [4], such as $\mathrm{MoS}_{2}, \mathrm{WS}_{2}, \mathrm{MoSe}_{2}$, and $\mathrm{WTe}_{2}$, have drawn people's attention for their non-zero band gaps, but the electron mobility is not efficiently high. Novel 2D materials of group $\mathrm{V}$ elements, such as black phosphorus [5-7], arsenene [8,9], antimonene [8,9], and bismuthene [10], are gradually theoretically proposed and experimentally realized. Although black phosphorus has a tuneable band gap from $0.3 \mathrm{eV}$ to $2.0 \mathrm{eV}$ and a high carrier mobility, it is not stable in the air. 
Haibo Zeng [8] et al. first predicted antimonene, as the monolayer format of antimony. It is a stable semiconductor with a tuneable band gap and high electrical mobility. Later more antimonene characteristics were predicted [11], such as the effect of interfacial distance [12], heterostructures [13], doping [14], thermal conductivity [15], optical properties [16,17], and so on. It has a wide indirect band gap and its band edge can be tuned by applying strain [8]. It can endure higher strain due to its van der Waals feature [18-20]. At the same time, antimonene growth has been attempted in a number of works [21-23] through molecular beam epitaxy (MBE) or other growth methods. Until now, beside $\beta$-antimonene, scientists have successfully grown honeycomb antimonene [24] and $\alpha$-antimonene [25] structures. As for the substrate choice, $\operatorname{GaAs}(111)$ [26], Ge(111) [27], c-plane sapphire [28], $\mathrm{Ag}(111)$, and h-BN $[24,29]$ have either been proposed or tried. However, experimental evidence is still insufficient in this area. For example, although antimonene has been made [30], the size is only in the nanometer level. Commonly available characterization methods, such as X-ray diffraction (XRD) and Raman spectra, cannot provide strong enough evidences. A distinctive Raman shift of antimonene is yet to be confirmed $[25,28,31]$.

Based on this background, we decided to grow antimonene on c-plane sapphire substrates. There are several reasons that we choose c-plane sapphire. Firstly, it is a stable, high-melting-point insulator, which does not contribute to the electrical characterization of the antimonene. Besides, it has a hexagonal structure that is similar to that of antimonene, and it can offer a $13.4 \%$ biaxial strain, according to theoretical calculation [8]. More importantly, van der Waals epitaxy has more opportunities to be achieved because of the absence of dangling bonds on the surface. During the growth study, we found that growth temperature played an important role in modulating the surface energy, therefore, to affect several key processes ", such as adsorption, cracking of the source molecules, and the antimonene nucleation. Moreover, we have used high resolution XRD not only to obtain the antimonene quality, but also to reveal the competition between $2 \mathrm{D}$ antimonene and three-dimensional (3D) antimony growth processes. In addition, choices of $\mathrm{Sb}_{2}$ or $\mathrm{Sb}_{4}$ molecules could affect the electrical properties of the grown samples. These results not only provide a potential method to grow antimonene on more commonly available substrates, but also critical parameters that can promote the $2 \mathrm{D}$ growths.

\section{Materials and Methods}

Antimonene was grown on c-plane sapphire substrate in an ultrahigh vacuum (UHV) chamber by molecular beam epitaxy (Veeco, GENxplor). The background pressure was $10^{-10}$ torr, the antimony beam equivalent pressure (BEP) was maintained between $5.67 \times 10^{-8}$ torr to $6.75 \times 10^{-8}$ torr (measured by a Beam Flux Monitor, BFM). Before the growth, we performed a substrate surface preparation in a thermal annealing furnace that was filled with oxygen. Annealing was performed at $1200{ }^{\circ} \mathrm{C}$ for $8 \mathrm{~h}$, which would yield a clear terraced surface. We use $0.5^{\circ}$ offcut angle c-plane sapphire that has about $40 \mathrm{~nm}$ wide terraces after annealing. While using an antimony (Sb) cracker cell, the forms of antimony molecules $\left(\mathrm{Sb}_{2}\right.$ or $\left.\mathrm{Sb}_{4}\right)$ can be controlled through changing the cracking zone temperature.

The electrical properties were measured on a Hall effect measurement system (Lakeshore, 8404) using van der Pauw method. The samples were cleaved to a square shape $(1 \mathrm{~cm} \times 1 \mathrm{~cm})$ and four indium electrodes were made on the corners. The DC mode was used in this experiment.

For the XRD measurements, a Bruker D8 high resolution XRD system was used. The radiation wavelength is $0.15406 \mathrm{~nm}\left(\mathrm{Cu} \mathrm{K}_{\alpha 1}\right)$. Continuous scan mode was used and the step sizes are $0.05^{\circ}$ for wide two-theta $\left(20^{\circ}-50^{\circ}\right)$ scan and $0.006^{\circ}$ for fine scans $\left(23^{\circ}-24.5^{\circ}, 28-29.5^{\circ}\right)$, and the integration time is $1 \mathrm{~s}$. A HORIBA HR800 Raman measurement system was used and the $10 \mathrm{~mW}$ laser wavelength is $488 \mathrm{~nm}$ with a spot size around $2 \mu \mathrm{m}$. The measurement range is $50 \mathrm{~cm}^{-1}-8000 \mathrm{~cm}^{-1}$, and the spectral resolution is $1 \mathrm{~cm}^{-1}$. Before each test, we used a silicon wafer to calibrate the Raman shift to exactly $521.7 \mathrm{~cm}^{-1}$ to ensure the accuracy of the following test results. 


\section{Results}

We have grown several groups of samples while using different growth conditions. Table 1 summarizes the growth details. The Group A samples were grown, while the $\mathrm{Sb}$ cracking temperature was set at $700{ }^{\circ} \mathrm{C}$ so the evaporated beam was composed with mostly $\mathrm{Sb}_{4}$ molecules. Group B samples were done with the $\mathrm{Sb}$ cracking temperature set at $1000{ }^{\circ} \mathrm{C}$, so $\mathrm{Sb}_{2}$ molecules were the primary source molecules in the evaporated beam (higher than $90 \%$ ). The electrical properties are only measured on the continuous films in our study, because most samples tend to form island structures, so the electrical performance (insulating or conducting) is hard to obtain. Similarly, it is difficult to have good XRD signals for the samples that are either too thin or no growth was done. In the following analysis, we have only used the results that are reliable and reproducible.

Table 1. Sample list with the growth parameters and properties. $\mathrm{Sb}_{4}$ molecules were used in the growth of group A samples and $\mathrm{Sb}_{2}$ molecules were used in the growth of group B samples. " $\backslash$ " means no data were obtained.

\begin{tabular}{cccccc}
\hline Sample No. & $\begin{array}{c}\text { Growth } \\
\text { Temperature }\left({ }^{\circ} \mathbf{C}\right)\end{array}$ & $\begin{array}{c}\text { Growth Time } \\
(\mathbf{m i n})\end{array}$ & Crystalline/Amorphous & $\begin{array}{c}\text { Electrical } \\
\text { Performance }\end{array}$ & $\begin{array}{c}\text { Effective } \\
\text { Thickness (nm) }\end{array}$ \\
\hline A-1 & 150 & 10 & amorphous & insulating & $57.81 \pm 4.38$ \\
B-1 & 150 & 10 & crystal & conducting & $\approx 15$ \\
A-2 & 200 & 30 & crystal & conducting & $32.01 \pm 2.08$ \\
A-3 & 225 & 30 & crystal & $\backslash$ & $32.11 \pm 9.40$ \\
A-4 & 250 & 30 & crystal & $\backslash$ & $10.48 \pm 3.73$ \\
A-5 & 265 & 30 & crystal & $\backslash$ & $1.33 \pm 0.37$ \\
A-6 & 270 & 30 & $\backslash$ & $\backslash$ & 0 \\
A-7 & 280 & 30 & $\backslash$ & \\
\hline
\end{tabular}

At the beginning, we tried to understand whether there is a difference between $\mathrm{Sb}_{2}$ and $\mathrm{Sb}_{4}$ in the growth. Figure 1a-c show the surface morphology. The antimony film of B-1 is a good conductor (more accurately, semimetal) according to the Hall measurement results (Table 2), but for A-1, it is an insulator. Another sample using $\mathrm{Sb}_{4}$ molecules (A-2) were grown under $200{ }^{\circ} \mathrm{C}$, and the electrical data can be seen in Table 2. It is obvious that sample A-2 is a semimetal with a carrier concentration in the $10^{20} / \mathrm{cm}^{3}$ level. The higher growth temperature for A-2 sample seems to introduce $\mathrm{Sb}_{2}$ molecules. Hence, we believe that there is a critical temperature $\left(\mathrm{T}_{\text {critical }}\right)$ that is between $150^{\circ} \mathrm{C}$ and $200{ }^{\circ} \mathrm{C}$, when $\mathrm{T}_{\text {growth }}<\mathrm{T}_{\text {critical, }}$, samples using $\mathrm{Sb}_{4}$ molecules are insulating and, on the other hand, conductive when $\mathrm{T}_{\text {growth }}>\mathrm{T}_{\text {critical }}$.
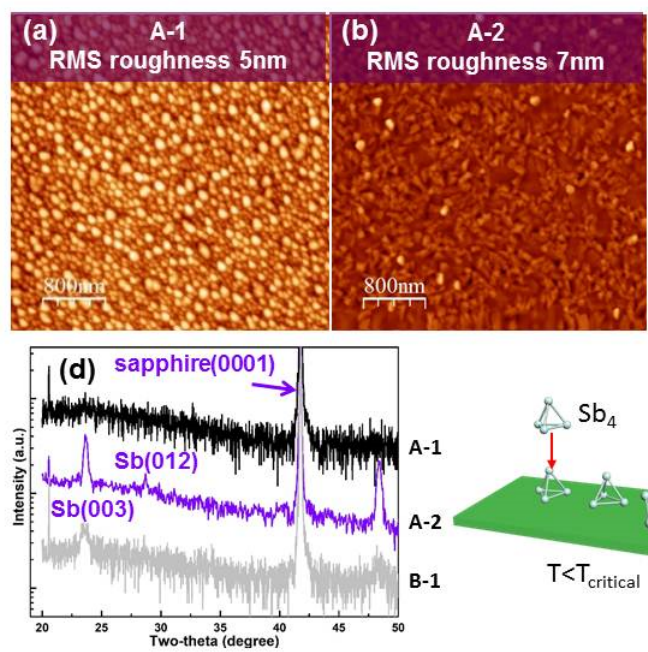

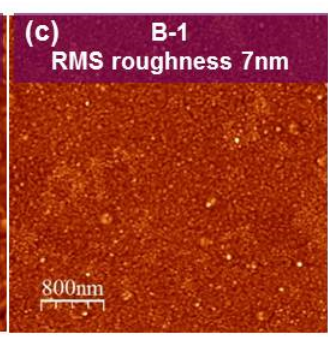

(e)

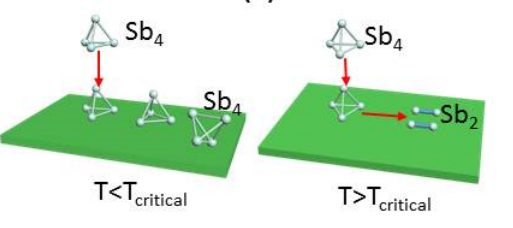

Figure 1. (a-c) Atomic force microscope (AFM) images of sample A-1, A-2, and B-1. (d) X-ray diffraction (XRD) diffractogram of sample A-1, A-2, and B-1. (e) Proposed cracking mechanism of $\mathrm{Sb}_{4}$ on the surface, showing the difference when $\mathrm{T}<\mathrm{T}_{\text {critical }}$ and $\mathrm{T}>\mathrm{T}_{\text {critical }}$. 
Table 2. Electrical properties of sample A-1, A-2, and B-1 resistances are measured from two orthogonal directions of a square-shaped sample.

\begin{tabular}{|c|c|c|c|c|c|c|}
\hline Sample No. & Carrier Type & $\begin{array}{l}R_{\text {sheet }} \\
(\Omega / \square)\end{array}$ & $\begin{array}{l}\text { Thickness } \\
\text { (nm) }\end{array}$ & $\begin{array}{c}\text { Carrier } \\
\text { Concentration } \\
\left(1 / \mathrm{cm}^{3}\right)\end{array}$ & $\begin{array}{c}\mu \\
\left(\mathrm{cm}^{2} / \mathrm{V} \cdot \mathrm{s}\right)\end{array}$ & $\begin{array}{c}\text { Resistances } \\
(\Omega) \\
\mathbf{R}_{\|} / \mathbf{R}_{\perp}\end{array}$ \\
\hline A-1 & 1 & 1 & $57.81 \pm 4.38$ & 1 & 1 & Insulating \\
\hline A-2 & $p$ & 75.04 & $32.01 \pm 2.08$ & $2.23 \times 10^{20}$ & 74.62 & $21.85 / 12.23$ \\
\hline B-1 & $p$ & 147.1 & $\approx 15$ & $4.39 \times 10^{20}$ & 64.44 & $39.81 / 26.08$ \\
\hline
\end{tabular}

The growth by $\mathrm{Sb}_{4}$ or $\mathrm{Sb}_{2}$ molecules might have different kinetic mechanisms, so the difference in electrical properties indicates the transformation of $\mathrm{Sb}_{4}$ to $\mathrm{Sb}_{2}$ on the sapphire surface above the critical temperature might introduce a different arrangement of the $\mathrm{Sb}_{2}$ molecules for the following nucleation. Most $\mathrm{Sb}_{4}$ molecules exist in a tetrahedral structure [32,33]. They are flattened into a planar structure when arriving the substrate surface. After that, they gradually change to a rectangle or distorted rectangle structure and, finally, they split into two $\mathrm{Sb}_{2}$ molecules, i.e., $\mathrm{Sb}_{4} \rightleftharpoons 2 \mathrm{Sb}_{2}$ (Figure 1e). When the growth temperature is below $\mathrm{T}_{\text {critical }}$, the surface energy cannot break the chemical bond of $\mathrm{Sb}_{4}$, so they will deposit as the tetrahedral form. Hence, it tends to grow more randomly, which can be proven by the XRD spectrum (Figure 1d). For A-1, it is amorphous, but, for B-1, it has a clear Sb (003) peak, which indicates the crystal structure. As for A-2, it has a stronger Sb (003) peak and other Sb peaks, which indicates it is polycrystalline feature.

From Figure 1d, we can see when we use $\mathrm{Sb}_{4}$ molecules, the $\mathrm{Sb}(012)$ peak can be observed, and, from literature, we know that the $\mathrm{Sb}(012)$ peak may be a feature of antimonene [28,31]. Accordingly, we chose $\mathrm{Sb}_{4}$ molecules to grow more antimonene samples. We tried to grow several samples with different growth temperatures, as shown in Figure $2 \mathrm{~b}-\mathrm{h}$. Figure $2 \mathrm{a}$ is the sapphire substrate that is used in this study. Before each growth, we undertook pretreatment on the c-plane sapphire substrate, so that terraces were formed on the surface. After annealing, $\mathrm{Sb}_{4}$ molecules were used to grow antimonene at different growth temperatures in the MBE chamber. Table 2 shows the details. From A-1 and A-2, we can see that the antimony covers the entire sapphire surface. However, when the growth temperature rises, the surface energy is getting higher, so the grown antimony will change to a triangular prism structure and cannot cover the entire surface. When the growth temperature rises to $265^{\circ} \mathrm{C}$, fewer antimony structure can be seen, but when the growth temperature rises to $270{ }^{\circ} \mathrm{C}$, it is hard to find the grown antimony by atomic force microscope(AFM) scan. Hence, we are convinced that $270{ }^{\circ} \mathrm{C}$ is too high for antimony molecules to stick and nucleate. A similar trend is observed by Raman spectroscopy measurements. Raman data (Figure 2i) show that Raman peaks can be seen at $110 \mathrm{~cm}^{-1}$ and $150 \mathrm{~cm}^{-1}$, which indicate the antimony structure [28]. Besides, under $265^{\circ} \mathrm{C}$, we can still see antimony Raman peaks and triangle column structure, but under $270{ }^{\circ} \mathrm{C}$, it shows no antimony. Accordingly, the highest nucleation temperature on an annealed sapphire substrate in our study is between $265^{\circ} \mathrm{C}$ and $270{ }^{\circ} \mathrm{C}$, which is similar to the value that was reported by Dura et al. [34,35].

In addition, we studied the stability of the grown samples. As we can see in Figure 2j,k, the AFM images on the same sample show that similar surface morphology is observed one year later, which means no apparent change occurred. Hence, we believe the antimonene samples are stable in the air for at least one year [18].

Figure 3a shows the XRD data that were measured from group A samples. It can be seen that, when the growth temperature is between $200{ }^{\circ} \mathrm{C}-250{ }^{\circ} \mathrm{C}$, the grown antimony has preferentially crystalized signals such as $\mathrm{Sb}(003)$ peak at $23.7^{\circ}$ and $\mathrm{Sb}(012)$ peak at $28.7^{\circ}$. Figure $3 \mathrm{~b}$ shows the zoomed-in diffractogram in that region. We can calculate the integrated area of each peak and easily obtain the ratio of $\mathrm{Sb}(012) / \mathrm{Sb}(003)$ as a function of the growth temperature. Since $\mathrm{Sb}(012)$ peak is the indicator of antimonene formation, this ratio can be used to find the preferred growth condition for antimonene. We define a term to represent the total crystalline content, which is calculated as the sum of the integrated areas of antimony (003) and (012) peaks, based on Figure 3b. As shown in Figure 3c,d 
(the blue curves), when growth temperature increases, the total crystalline content on the sapphire surface decreases in the growth window until zero. It is worth mentioning that all of the XRD scans are symmetric scans on the sapphire (0001) so only the crystal planes that are parallel to the surface can be measured. This means the total crystalline content is underestimated and the detected signals indicate the coherence of the crystals that are likely to have been epitaxially grown on the substrate. We also introduce an effective adsorption coefficient to describe the adsorption process of $\mathrm{Sb}_{4}$ molecules on c-plane sapphire, as shown in Figure 3c (the red curve). The effective adsorption coefficient is calculated while using the ratio of the effective thickness at all growth temperatures (Table 1 ) and the effective thickness of $150{ }^{\circ} \mathrm{C}$. We assume that, under $150{ }^{\circ} \mathrm{C}$, the effective adsorption coefficient is 1 , because of the low vapor pressure and high sticking coefficient of $\mathrm{Sb}$ at this temperature [36]. Calculation details can be found in the Supplementary Materials.
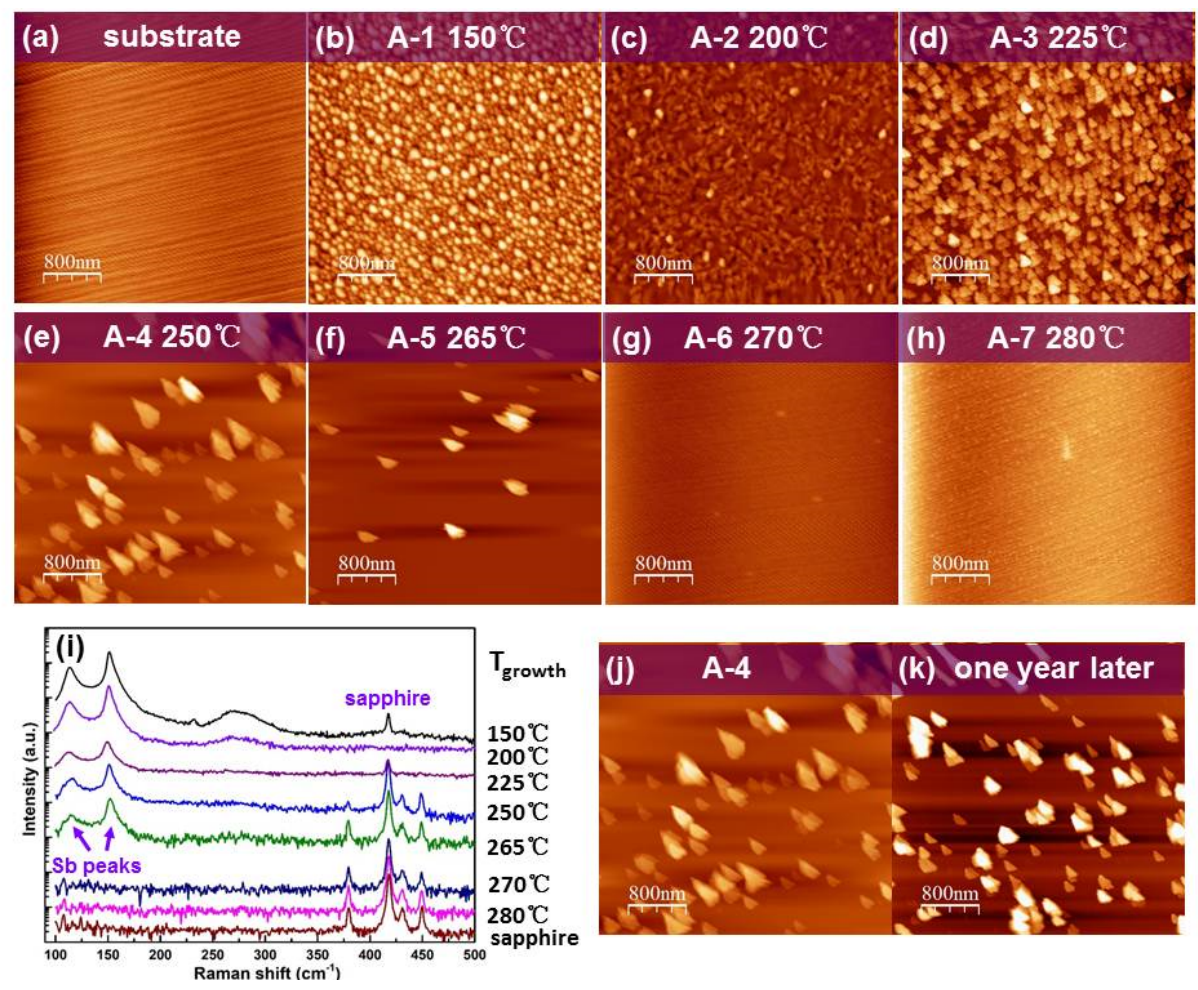

Figure 2. (a-h) AFM images of a c-plane sapphire substrate and sample A-1 to A-7, showing the surface morphology change due to the temperature increment. (i) Raman spectra of all the group A $\left(\mathrm{Sb}_{4}\right)$ samples. Peaks at $350 \mathrm{~cm}^{-1}$ to $450 \mathrm{~cm}^{-1}$ are from the c-plane sapphire, $110 \mathrm{~cm}^{-1}$ and $150 \mathrm{~cm}^{-1}$ are the two signature vibrational modes of antimony. $(\mathbf{j}, \mathbf{k})$ AFM images of antimonene, being characterized immediately after the sample is taken out of the vacuum and one year later.

We tried to fit the $\mathrm{Sb}(003)$ peak and $\mathrm{Sb}$ (012) peak using a multi-order Gauss function to further determine the antimonene percentage more quantitatively in the grown samples. Subsequently, we calculated the integrated area of each peak and got the ratio of $\mathrm{Sb}(012) /[\mathrm{Sb}(003)+\mathrm{Sb}(012)]$ to estimate the antimonene percentage in the crystalline contents. Based on this calculation, we draw the phase diagram of antimonene formation and crystalline content as a function of the growth temperature on c-plane sapphire, as shown in Figure 3d. We can see that, when the growth temperature is between $220^{\circ} \mathrm{C}-260{ }^{\circ} \mathrm{C}$, there is a clear trend showing the antimonene content increases, which exceeds 50 percent. However, during the same temperature region, the total crystalline content decreases, which indicates that the reduction of effective adsorption limits the yield of crystal growth. This competing mechanism makes the preferential growth of antimonene very difficult. When the growth temperature is either between $200^{\circ} \mathrm{C}-220^{\circ} \mathrm{C}$ or $260^{\circ} \mathrm{C}-270^{\circ} \mathrm{C}$, the antimonene content becomes 
lower than 50 percent. When it is below $200{ }^{\circ} \mathrm{C}$, the deposited antimony on sapphire is amorphous and, when it is higher than $270^{\circ} \mathrm{C}$, no growth is observed, which is mainly due to the low sticking coefficient. This phase diagram provides a competing mechanism during the antimony deposition on sapphire. Unfortunately, the growth window is quite small and it cannot yield pure antimonene. We think a possible growth process is that $\mathrm{Sb}_{4}$ molecules need to be adsorbed first and then cracked to $\mathrm{Sb}_{2}$ on the surface since antimonene is a $2 \mathrm{D}$ growth mechanism and antimony is a $3 \mathrm{D}$ growth mechanism. Subsequently, the $\mathrm{Sb}_{2}$ molecules need enough surface energy to rearrange and grow in $2 \mathrm{D}$. A mixture of $3 \mathrm{D}$ and $2 \mathrm{D}$ formats of antimony growth occurs since both $\mathrm{Sb}_{4}$ and $\mathrm{Sb}_{2}$ molecules are present on the surface and the formation energies of 3D antimony and 2D antimonene are close. Within the growth window, 2D growth can be promoted by a higher surface temperature, which makes us believe that enough surface energy is the key to enhance van der Waals epitaxy over a typical 3D growth.

One of the characters of antimonene is that it can be grown on a substrate by van der Waals epitaxy, but the energy of van der Waals force is at least one order of magnitude smaller than that of a chemical bond, which makes antimonene growth more challenging. In addition to surface energy, which can be tuned by controlling growth temperature, there are some other methods that may improve the probability of van der Waals epitaxy. For example, we can try to enlarge the distance between antimonene and substrate by depositing larger radius atoms (such as bismuth) as surfactant. In addition, the substrate surface can be passivated by using hydrogen atoms. Graphene or h-BN also can be used to be inserted between the grown antimonene and the substrate, since they are more compatible with materials through van der Waals force, which was experimentally proven. Besides, methods such as how to regulate the formation energy of antimonene and antimony to choose the optimized growth conditions are also useful and prospective, but they need further exploration.
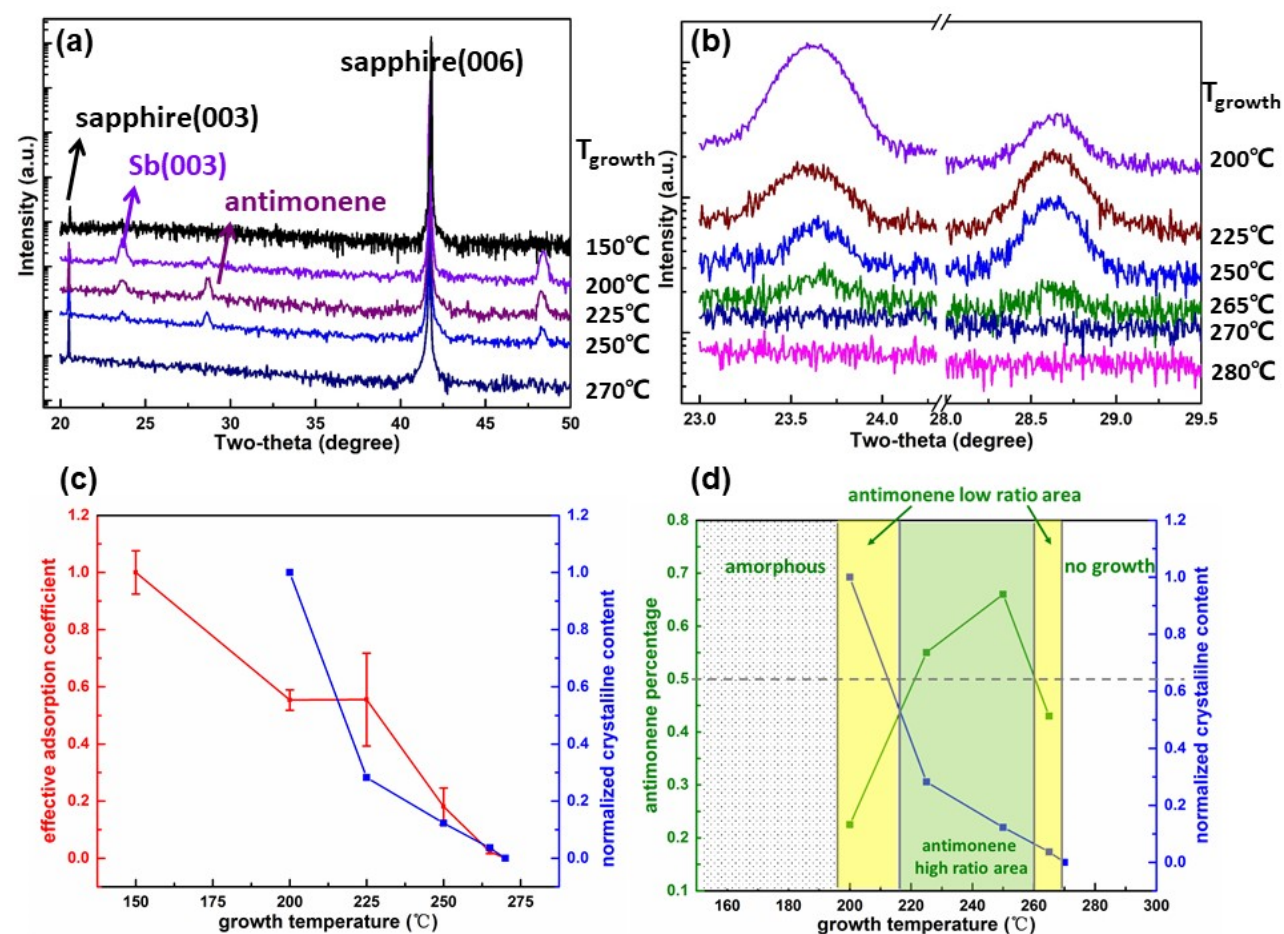

(d)

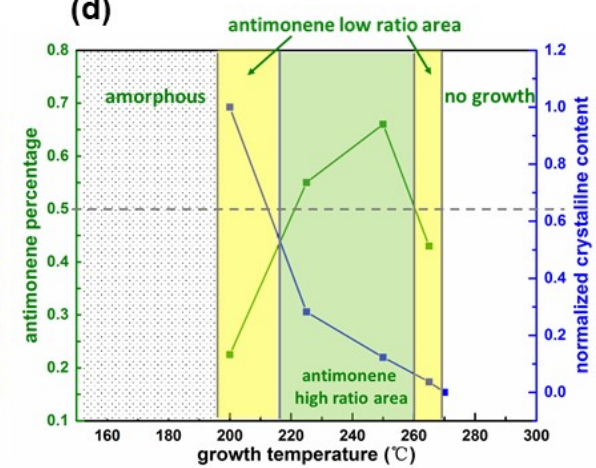

Figure 3. (a) XRD diffractograms of some group A samples using $\mathrm{Sb}_{4}$. (b) XRD diffractograms between $23^{\circ}-29.5^{\circ}$, showing the antimonene features. (c) The effective adsorption coefficient of $\mathrm{Sb}_{4}$ and normalized crystalline contents (integrated area of antimony (003) and (012) peaks. The maximum is 1 after normalized) as a function of the growth temperature. (d) Phase diagram showing the comparison of the antimonene formation and total crystalline contents as a function of growth temperature, indicating the competing mechanisms between the adsorption and nucleation of antimony molecules. 


\section{Conclusions}

In conclusion, we have shown that large-area antimonene can be grown on c-plane sapphire and it can be characterized by XRD and Raman spectra. The growth window is small in terms of growth temperature, which is $200{ }^{\circ} \mathrm{C}-265{ }^{\circ} \mathrm{C}$. Below $200{ }^{\circ} \mathrm{C}$, the deposited antimony is amorphous andthere is no growth higher than $270^{\circ} \mathrm{C}$. We further refined the growth window for antimonene, which is $220^{\circ} \mathrm{C}-260^{\circ} \mathrm{C}$ and discussed the competing mechanism between $2 \mathrm{D}$ and $3 \mathrm{D}$ growths. Besides, we discussed the nucleation temperature of antimony growth and the critical temperature to explain the electrical characteristics. This work provides a potential method to grow large area antimonene on sapphire, which is important for further characterizations of thermal, optical, and electrical properties.

Supplementary Materials: The following are available online at http://www.mdpi.com/2076-3417/10/2/639/s1, Figure S1: AFM image of sample A-1 and the height profile cross the film and the substrate, Figure S2: AFM image of sample A-4 and the profile of one line scan. (unit: $\mathrm{nm}$ ).

Author Contributions: Conceptualization, M.G., K.Z. and H.L.; methodology, M.G., Y.D., S.X., Y.W. and C.L.; investigation, M.G. and K.Z.; writing-original draft preparation, M.G.; writing-review and editing, M.G., C.L. and H.L.; supervision, M.-H.L., H.L. and Y.-F.C. All authors have read and agreed to the published version of the manuscript.

Funding: The National Key R\&D Program of China (2018YFA0306200, 2017YFA0303702), the National Natural Science Foundation of China (Grant No. 51732006, No. 11890702, No. 51721001, No. 51702153), Natural Science Foundation of Jiangsu Province (BK20160627), the Thousand Talents Program and Jiangsu Entrepreneurship and Innovation Program.

Acknowledgments: M.G. thanks X.W. and his students for the help in Raman measurements. The authors acknowledge the support from the National Key R\&D Program of China (2018YFA0306200, 2017YFA0303702), the National Natural Science Foundation of China (Grant No. 51732006, No. 11890702, No. 51721001, No. 51702153), Natural Science Foundation of Jiangsu Province (BK20160627), the Thousand Talents Program and Jiangsu Entrepreneurship and Innovation Program.

Conflicts of Interest: The authors declare no conflict of interest.

\section{References}

1. Geim, A.K.; Novoselov, K.S. The rise of graphene. Nat. Mater. 2007, 6, 183-191. [CrossRef]

2. Xu, X.; Zhang, Z.; Dong, J.; Yi, D.; Niu, J.; Wu, M.; Lin, L.; Yin, R.; Li, M.; Zhou, J.; et al. Ultrafast epitaxial growth of metre-sized single-crystal graphene on industrial Cu foil. Sci. Bull. 2017, 62, 1074-1080. [CrossRef]

3. Xu, X.; Zhang, Z.; Qiu, L.; Zhuang, J.; Zhang, L.; Wang, H.; Liao, C.; Song, H.; Qiao, R.; Gao, P.; et al. Ultrafast growth of single-crystal graphene assisted by a continuous oxygen supply. Nat. Nanotechnol. 2016, 11, 930-935. [CrossRef]

4. Li, H.; Li, Y.; Aljarb, A.; Shi, Y.; Li, L.-J. Epitaxial Growth of Two-Dimensional Layered Transition-Metal Dichalcogenides: Growth Mechanism, Controllability, and Scalability. Chem. Rev. 2017, 118, 6134-6150. [CrossRef] [PubMed]

5. Li, L.; Yu, Y.; Ye, G.J.; Ge, Q.; Ou, X.; Wu, H.; Feng, D.; Chen, X.H.; Zhang, Y. Black phosphorus field-effect transistors. Nat. Nanotechnol. 2014, 9, 372-377. [CrossRef] [PubMed]

6. Koenig, S.P.; Doganov, R.A.; Schmidt, H.; Neto, A.H.C.; Oezyilmaz, B. Electric field effect in ultrathin black phosphorus. Appl. Phys. Lett. 2014, 104, 103106. [CrossRef]

7. Rodin, A.S.; Carvalho, A.; Castro Neto, A.H. Strain-induced gap modification in black phosphorus. Phys. Rev. Lett. 2014, 112, 176801. [CrossRef] [PubMed]

8. Zhang, S.; Yan, Z.; Li, Y.; Chen, Z.; Zeng, H. Atomically thin arsenene and antimonene: Semimetal-semiconductor and indirect-direct band-gap transitions. Angew. Chem. 2015, 127, 3155-3158. [CrossRef]

9. Pumera, M.; Sofer, Z. 2D Monoelemental Arsenene, Antimonene, and Bismuthene: Beyond Black Phosphorus. Adv. Mater. 2017, 29, 1605299. [CrossRef]

10. Reis, F.; Li, G.; Dudy, L.; Bauernfeind, M.; Glass, S.; Hanke, W.; Thomale, R.; Schäfer, J.; Claessen, R. Bismuthene on a $\mathrm{SiC}$ substrate: A candidate for a high-temperature quantum spin Hall material. Science 2017, 357, 287-290. [CrossRef] 
11. Zhang, S.; Xie, M.; Li, F.; Yan, Z.; Li, Y.; Kan, E.; Liu, W.; Chen, Z.; Zeng, H. Semiconducting Group?15 Monolayers: A Broad Range of Band Gaps and High Carrier Mobilities. Angew. Chem. 2016, 128, 1698-1701. [CrossRef]

12. Zhang, F.; Li, W.; Dai, X. Effects of interlayer coupling on the electronic structures of antimonene/graphene van der Waals heterostructures. Superlattices Microstruct. 2016, 100, 826-832. [CrossRef]

13. Lu, H.; Gao, J.; Hu, Z.; Shao, X. Biaxial strain effect on electronic structure tuning in antimonene-based van der Waals heterostructures. RSC Adv. 2016, 6, 102724-102732. [CrossRef]

14. Xie, M.; Zhang, S.; Cai, B.; Zou, Y.; Zeng, H. N- and p-type doping of antimonene. RSC Adv. 2016, 6, 14620-14625. [CrossRef]

15. Wang, S.; Wang, W.; Zhao, G. Thermal transport properties of antimonene: An ab initio study. Phys. Chem. Chem. Phys. 2016, 18, 31217-31222. [CrossRef]

16. Huo, C.; Sun, X.; Yan, Z.; Song, X.; Zhang, S.; Xie, Z.; Liu, J.; Ji, J.; Jiang, L.; Zhou, S.; et al. Retraction of "Few-Layer Antimonene: Large Yield Synthesis, Exact Atomical Structure, and Outstanding Optical Limiting". J. Am. Chem. Soc. 2017, 139, 35688. [CrossRef]

17. Tsai, H.-S.; Chen, C.-W.; Hsiao, C.-H.; Ouyang, H.; Liang, J.-H. The advent of multilayer antimonene nanoribbons with room temperature orange light emission. Chem. Commun. 2016, 52, 8409-8412. [CrossRef]

18. Koma, A. Van der Waals epitaxy-A new epitaxial growth method for a highly lattice-mismatched system. Thin Solid Films 1992, 216, 72-76. [CrossRef]

19. Koma, A. Van der Waals epitaxy for highly lattice-mismatched systems. J. Cryst. Growth 1999, 201, $236-241$. [CrossRef]

20. Koma, A.; Sunouchi, K.; Miyajima, T. Summary Abstract: Fabrication of ultrathin heterostructures with van der Waals epitaxy. J. Vac. Sci. Technol. B Microelectron. Nanom. Struct. 1985, 3, 724. [CrossRef]

21. Wu, X.; Shao, Y.; Liu, H.; Feng, Z.; Ibrahim, K.; Wang, Y.; Sun, J.; Zhu, S.; Du, S.; Shi, Y.; et al. Epitaxial Growth and Air-Stability of Monolayer Antimonene on PdTe 2. Adv. Mater. 2016, 29, 1605407. [CrossRef] [PubMed]

22. Gibaja, C.; Rodriguez-San-Miguel, D.; Ares, P.; Gómez-Herrero, J.; Varela, M.; Gillen, R.; Maultzsch, J.; Hauke, F.; Hirsch, A.; Abellan, G. Few-Layer Antimonene by Liquid-Phase Exfoliation. Angew. Chem. Int. Ed. 2016, 55, 14345-14349. [CrossRef] [PubMed]

23. Ji, J.; Song, X.; Liu, J.; Yan, Z.; Huo, C.; Zhang, S.; Su, M.; Liao, L.; Wang, W.; Ni, Z.; et al. Two-dimensional antimonene single crystals grown by van der Waals epitaxy. Nat. Commun. 2016, 7, 13352. [CrossRef] [PubMed]

24. Shao, Y.; Liu, Z.-L.; Cheng, C.; Wu, X.; Liu, H.; Liu, C.; Wang, J.-O.; Zhu, S.-Y.; Wang, Y.-Q.; Shi, D.-X.; et al. Epitaxial Growth of Flat Antimonene Monolayer: A New Honeycomb Analogue of Graphene. Nano Lett. 2018, 18, 2133-2139. [CrossRef]

25. Shi, Z.Q.; Li, H.; Yuan, Q.Q.; Song, Y.H.; Lv, Y.Y.; Shi, W.; Jia, Z.-Y.; Gao, L.; Chen, Y.-B.; Zhu, W.; et al. Van der Waals heteroepitaxial growth of monolayer $\mathrm{Sb}$ in puckered honeycomb structure. arXiv 2019, arXiv:1903.00641. [CrossRef]

26. Wang, N.; Cao, D.; Wang, J.; Liang, P.; Chen, X.; Shu, H. Interface effect on electronic and optical properties of antimonene/GaAs van der Waals heterostructures. J. Mater. Chem. C 2017, 5, 9687-9693. [CrossRef]

27. Fortin-Deschênes, M.; Waller, O.; Menteş, T.O.; Locatelli, A.; Mukherjee, S.; Genuzio, F.; Levesque, P.L.; Hébert, A.; Martel, R.; Moutanabbir, O. Synthesis of Antimonene on Germanium. Nano Lett. 2017, 17, 4970-4975. [CrossRef]

28. Chen, H.-A.; Sun, H.; Wu, C.-R.; Wang, Y.-X.; Lee, P.-H.; Pao, C.-W.; Lin, S.-Y. Single-Crystal Antimonene Films Prepared by Molecular Beam Epitaxy: Selective Growth and Contact Resistance Reduction of the 2D Material Heterostructure. ACS Appl. Mater. Interfaces 2018, 10, 15058-15064. [CrossRef]

29. Zhang, S.; Ma, Y.; Peng, R.; Huang, B.; Dai, Y. Ideal inert substrates for planar antimonene: h-BN and hydrogenated $\mathrm{SiC}(0001)$. Phys. Chem. Chem. Phys. 2018. [CrossRef]

30. Wang, X.; He, J.; Zhou, B.; Zhang, Y.; Wu, J.; Hu, R.; Liu, L.; Song, J.; Qu, J. Bandgap-Tunable Preparation of Smooth and Large Two-Dimensional Antimonene. Angew. Chem. 2018, 57, 8668-8673. [CrossRef]

31. Ares, P.; Aguilar-Galindo, F.; Rodríguez-San-Miguel, D.; Aldave, D.A.; Díaz-Tendero, S.; Alcamí, M.; Martín, F.; Gómez-Herrero, J.; Zamora, F. Mechanical Isolation of Highly Stable Antimonene under Ambient Conditions. Adv. Mater. 2016, 28, 6332-6336. [CrossRef] 
32. Martínez-Guerra, E.; Falkenberg, G.; Johnson, R.L.; Takeuchi, N. Adsorption ofSb4on Ge(001) and Si(001) surfaces: Scanning tunneling microscopy and first-principles calculations. Phys. Rev. B 2006, 73, 075302. [CrossRef]

33. Kaxiras, E. Interplay of Strain and Chemical Bonding in Surfactant Monolayers. EPL Europhys. Lett. 1993, 21, 685-690. [CrossRef]

34. Golding, T.D.; Dura, J.A.; Wang, W.C.; Vigliante, A.; Moss, S.C.; Chen, H.C.; Miller, J.H.; Hoffman, C.A.; Meyer, J.R. Sb/GaSb heterostructures and multilayers. Appl. Phys. Lett. 1993, 63, 1098-1100. [CrossRef]

35. Dura, J.A.; Vigliante, A.; Golding, T.D.; Moss, S.C. Epitaxial growth of Sb/GaSb structures: An example of V/III-V heteroepitaxy. J. Appl. Phys. 1995, 77, 21-27. [CrossRef]

36. Margrave, J.H. The Characterization of High Temperature Vapors; John Wiley \& Sons: New York, NY, USA, 1967.

(C) 2020 by the authors. Licensee MDPI, Basel, Switzerland. This article is an open access article distributed under the terms and conditions of the Creative Commons Attribution (CC BY) license (http://creativecommons.org/licenses/by/4.0/). 